\section{Adaptive optics and the eye (super resolution OCT)}

DT Miller, OP Kocaoglu, Q Wang and S Lee

\begin{abstract}
The combination of adaptive optics (AO) and optical coherence tomography (OCT) was first reported 8 years ago and has undergone tremendous technological advances since then. The technical benefits of adding AO to OCT (increased lateral resolution, smaller speckle, and enhanced sensitivity) increase the imaging capability of OCT in ways that make it well suited for three-dimensional (3D) cellular imaging in the retina. Today, AO-OCT systems provide ultrahigh $3 \mathrm{D}$ resolution $\left(3 \times 3 \times 3 \mu \mathrm{m}^{3}\right)$ and ultrahigh speed (up to an order of magnitude faster than commercial OCT). AO-OCT systems have been used to capture volume images of retinal structures, previously only visible with histology, and are being used for studying clinical conditions. Here, we present representative examples of cellular structures that can be visualized with AO-OCT. We overview three studies from our laboratory that used ultrahigh-resolution AO-OCT to measure the cross-sectional profiles of individual bundles in the retinal nerve fiber layer; the diameters of foveal capillaries that define the terminal rim of the foveal avascular zone; and the spacing and length of individual cone photoreceptor outer segments as close as $\mathbf{0 . 5 ^ { \circ }}$ from the fovea center. Eye (2011) 25, 321-330; doi:10.1038/eye.2011.1
\end{abstract}

Keywords: optical coherence tomography; adaptive optics; retinal nerve fibers; retinal capillaries; foveal avascular zone; photoreceptors

\section{Introduction}

The last 20 years have experienced extraordinary advances in optical technology to image the posterior segment of the eye non-invasively and at high resolution. Two of the most impactful technological advancements over this period have arguably been optical coherence tomography (OCT) and adaptive optics (AO). OCT - a low-coherence interferometric technique first reported in 1991 - provides unprecedented, micron-scale axial resolution $(<3 \mu \mathrm{m})$ and sensitivity to detect reflections from essentially any retinal layer, including those that are highly transparent. ${ }^{1,2}$ OCT has become a standard diagnostic tool for evaluating health of the posterior segment, as, for example, for macular holes, central serous chorioretinopathy, agerelated macular degeneration (ARMD), macular edema, diabetic retinopathy, and glaucoma.

Unlike OCT, AO is not an imaging modality, but rather a technology that can be used in combination with imaging modalities to improve their performance. AO works by measuring and correcting ocular aberrations at real time. Its benefit is greatest when the pupil is large $(>6 \mathrm{~mm})$, which has the added benefit of minimizing the blurring effects caused by diffraction. Correction of ocular imperfections across a large pupil results in unprecedented lateral resolution ( $2-3 \mu \mathrm{m})$, sufficient for resolving individual cells en face. ${ }^{3-5} \mathrm{AO}$ was originally developed for ground-based telescopes to remove atmospheric blur and later incorporated into retinal imaging in the mid-1990s. AO has become a valuable tool in vision research for studying structure and function of the microscopic retina, as well as the onset and progression of disease. Substantial maturation of the underlying technologies for use with the eye (eg, deformable mirrors and wavefront sensors) coupled with an expanding role for visualizing the microscopic retina suggest that commercialization for ophthalmic use is imminent.

$\mathrm{AO}$ and OCT provide complementary strengths in resolution (see Figure 1). AO provides improved lateral resolution and OCT provides improved axial resolution. Given the success of these technologies individually, there has been considerable effort by several

\author{
School of Optometry, \\ Indiana University, \\ Bloomington, IN, USA \\ Correspondence: DT Miller, \\ School of Optometry, \\ Indiana University, \\ 800 East Atwater Avenue, \\ Bloomington, IN 47405, \\ USA \\ Tel: + 8128557613 ; \\ Fax: + 8128557045 \\ E-mail: dtmiller@ \\ indiana.edu
}

Received: 3 November 2010 Accepted: 21 December 2010 

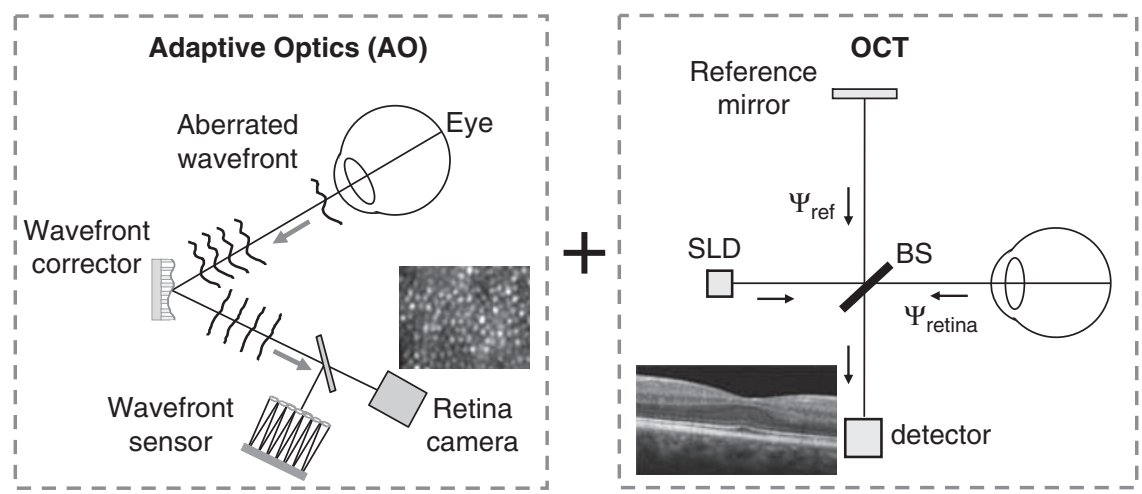

Figure 1 Micron-level resolution in all three dimensions realized by combining AO and OCT technologies. OCT uses low-coherence interferometry to achieve high axial resolution. AO measures the ocular aberrations with a wavefront sensor and corrects for them with a wavefront corrector to achieve the complementary high lateral resolution. In all current AO-OCT systems, AO is integrated into the sample channel of the OCT. In two early embodiments, $\mathrm{AO}$ was integrated into the detection channel.

research groups over the last decade to combine these technologies for high-resolution, three-dimensional (3D) imaging of the retina. Imaging at this level holds considerable promise to improve the research and clinical utility of OCT: for probing structure and function of the microscopic retina, and for earlier detection, more precise diagnosis, and improved treatment monitoring of posterior segment disease.

In this article, we present a brief overview of AO-OCT development, the technical benefits of adding $\mathrm{AO}$ to $\mathrm{OCT}$, and the use of AO-OCT to image the microscopic retina. Three representative cellular structures (retinal nerve fiber bundles (RNFBs), foveal capillaries, and cone photoreceptors) are selected and examples are given to demonstrate what $\mathrm{AO}-\mathrm{OCT}$ can reveal about them.

\section{AO-OCT technology}

$\mathrm{AO}$ has been demonstrated for the correction of higherorder aberrations in the three major types of ophthalmic imaging modalities: flood-illumination ophthalmoscope (fundus camera), scanning laser ophthalmoscope (SLO), and OCT. It was first demonstrated in the floodillumination ophthalmoscope in 1997 for imaging of cone photoreceptors and vision improvement. ${ }^{3}$ The concept of AO in an SLO was first proposed by Dreher et al, ${ }^{6}$ but not fully implemented with a wavefront sensor - which had not yet been developed for the eye - until the work by Roorda et al. ${ }^{7}$ Unlike the flood-illumination and SLO modalities that have effectively one principle design configuration, OCT embodies several fundamentally different ones that fall into two broad categories: time domain and spectral (or Fourier) domain. These domains refer to the temporal and spectral detection of the OCT signal, respectively, details of which can be found elsewhere. ${ }^{8}$ Over the last
8 years, all the major OCT designs have been demonstrated with AO. AO-OCT combinations include time-domain en face ( $x y$ ) flood-illumination OCT using an aerial $\mathrm{CCD},{ }^{9}$ time-domain tomographic scanning $(x z)$ ultrahigh-resolution $\mathrm{OCT}_{1}^{10}$ time-domain en face scanning $\mathrm{OCT}_{1}{ }^{11,12}$ high-resolution spectral-domain $\mathrm{OCT}_{1}{ }^{13-17}$ ultrahigh-resolution spectral-domain $\mathrm{OCT}_{1}^{18-22}$ and, more recently, swept source OCT. ${ }^{23}$

AO provides three technical benefits for OCT that improve the visualization and detection of microscopic structures in the retina: (1) increased lateral resolution, (2) reduced speckle size (granular artifacts), and (3) increased sensitivity to weak reflections. The addition of AO typically increases the lateral resolution by roughly five times over commercial OCT. Of course, to take advantage of this resolution requires increased A-scan sampling so that the closeness of the individual A-scans does not limit lateral resolution. Current state-of-the-art ultrahigh-resolution, $\mathrm{AO}$, spectraldomain OCT (UHR-AO-OCT) has an isotropic 3D resolution of $3 \times 3 \times 3 \mu \mathrm{m}^{3}$ in the retinal tissue. For comparison, Figure 2 illustrates the size of this resolution element (smallest black symbol) relative to that of OCT without $\mathrm{AO}$ and to that of the other two imaging modalities with and without AO. As shown, UHR-AO-OCT provides the best resolving capability and the only instrument able to resolve cells in all three dimensions.

A second benefit of $\mathrm{AO}$ is reduced speckle size. The interferometric nature of OCT generates high-contrast speckle that permeates the entire OCT image and masks structural details, especially those at the resolution limit of the system. As lateral speckle size is inversely related to pupil (beam) diameter, the larger pupil size afforded with $\mathrm{AO}(>6 \mathrm{~mm})$ reduces the lateral dimension of speckle by roughly five times and the corresponding 


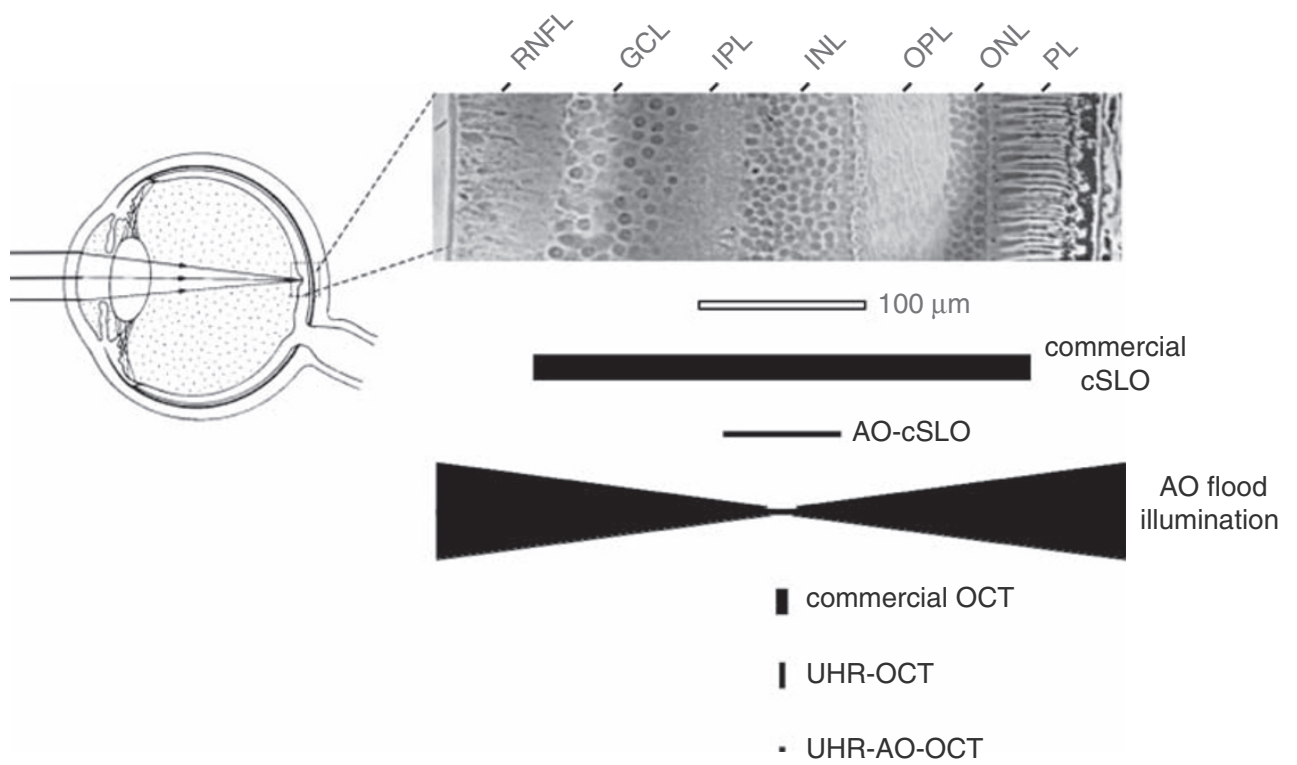

Figure 2 Comparison of (top) cell size in a histological cross section of the human retina with (bottom) the resolving capability of the major types of retinal imaging modalities with and without AO. The vertical and horizontal dimensions of the solid black symbols denote, respectively, the lateral and axial resolution of the instruments. Examples shown include the commercial confocal scanning laser ophthalmoscope (cSLO), confocal scanning laser ophthalmoscope with adaptive optics (AO-cSLO), flood illumination with adaptive optics, commercial OCT, ultrahigh-resolution OCT (UHR-OCT), and ultrahigh-resolution OCT with adaptive optics (UHRAO-OCT). GCL, ganglion cell layer; IPL, inner plexiform layer; INL, inner nuclear layer; OPL, outer plexiform layer; ONL, outer nuclear layer; PL, photoreceptor layer; RNFL, retinal nerve fiber layer. The histological cross section is from ref.25, reproduced with permission from the Royal Society of London.

speckle volume by 25 times, compared with that of commercial OCT. This substantial reduction noticeably improves the appearance of the OCT image when magnified, as illustrated by the commercial OCT and UHR-AO-OCT examples of Figures 3 and 4. Figure 3 shows a typical Heidelberg Spectralis image (setting: average of seven scans) acquired on a 62-year-old subject. At the magnification shown, the figure B-scan has a photographic-like quality that shows clear delineation of the various neuronal layers. Speckle appears largely absent and certainly inconsequential for examining the retinal features visible in the image. Magnification necessary to view the microscopic features (see Figure 4, top), however, presents a very different view. Speckle and image blur are now obvious artifacts and mask retinal features that would otherwise be present in the image. This is particularly evident by comparing the Spectralis B-scan (Figure 4, top) with the UHR-AO-OCT B-scan (Figure 4, bottom), which is of essentially the same patch of retina. Although maximum lateral resolution and structural detail are confined to the depth of focus $(<100 \mu \mathrm{m})$ that straddles the focus plane (which is at the retinal nerve fiber layer (RNFL) in the figure), speckle is noticeably smaller throughout the entire image. Thus, most of the visual difference between the commercial OCT and UHR-AO-OCT B-scans is due to differences in speckle size. Note that the lateral dimension of speckle is five times smaller (because of $\mathrm{AO}$ ) and the axial dimension of speckle is roughly two times smaller (because of UHR-OCT).

A third benefit is the increased sensitivity to weak reflections that results from the larger pupil afforded by $\mathrm{AO}$ and that captures more of the retinal reflectance. We have measured this improvement to be $4-8 \mathrm{~dB}$, depending on which retinal layer the focus of the AO-OCT instrument is placed. ${ }^{24,25}$

\section{Imaging the retina with AO-OCT}

AO-OCT systems have been used to capture volume images of retinal structures that were previously only visible with histology. Examples include the bundles within the RNFL; retinal microvasculature such as the capillaries that form the rim of the foveal avacular zone (FAZ); microstructures in the ganglion cell layer and Henle's fiber layer; the 3D photoreceptor mosaic; retinal pigment epithelium; and the tiny pores of the lamina cribrosa of the optical nerve. AO-OCT is being used for studying a number of clinical conditions, including ARMD, hereditary retinal dystrophies, retinopathy of prematurity, ${ }^{26}$ and optic neuropathies. ${ }^{27,28}$ 

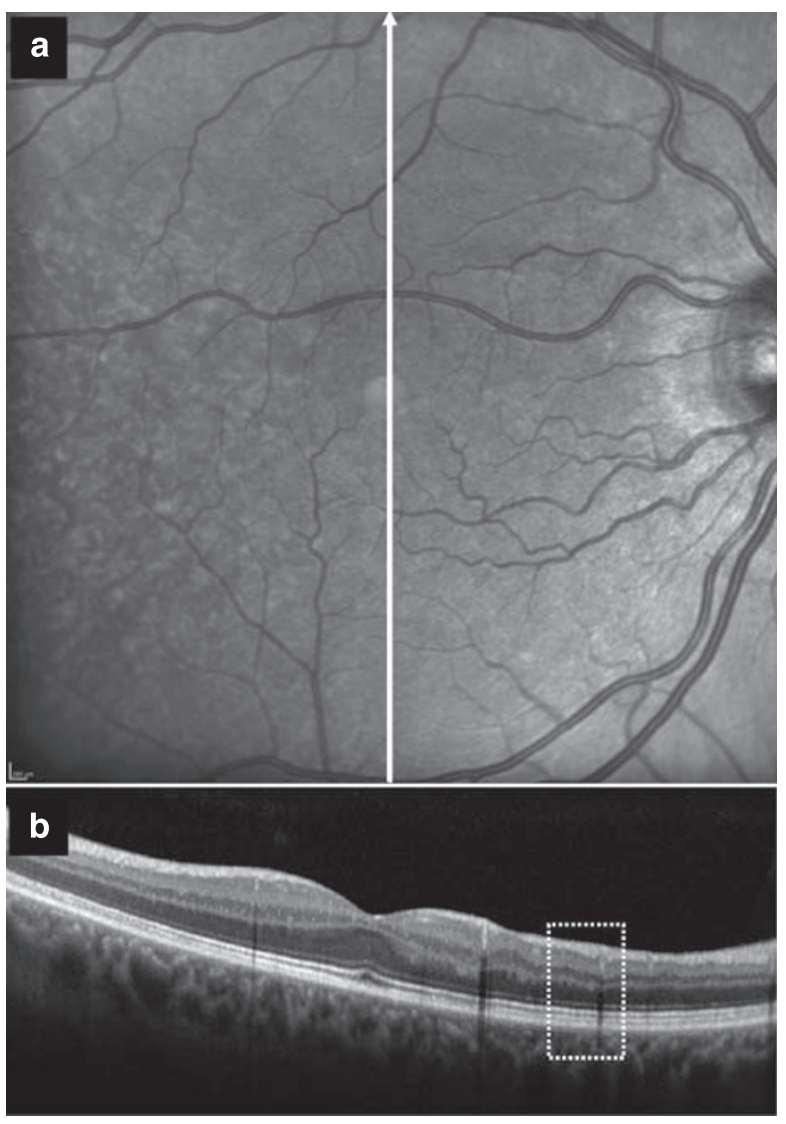

Figure 3 Heidelberg Spectralis of the right eye of a 62-year-old subject showing (a) $20^{\circ}$ fundus view and (b) vertical B-scan that traverses the fovea. Shown is the average of seven B-scans (Spectralis setting). Region highlighted by the white, dashed box ( $3^{\circ}$ wide) is magnified and displayed in Figure 4.

Figure 5 provides an illustrative example of the types of retinal microstructures that can be visualized within a given volume of retinal tissue. The volume shown is a composite made by re-imaging the same retinal patch, with focus systematically shifted to different depths, in this way preserving the high image quality provided by $\mathrm{AO}$ for the retinal layer of interest. Extracted en face slices (from top to bottom) are shown of individual RNFBs, microstructures in the ganglion cell layer, retinal capillaries, and outer segments of cone photoreceptors.

As suggested by the figure, $\mathrm{AO}-\mathrm{OCT}$ has already been applied to many of the retinal layers. Much of the motivation behind these studies has been to determine exactly what can be visualized and what new information can be obtained with AO-OCT. Of course, in parallel, substantial engineering has been necessary to apply and optimize these complex technologies to the eye. Described below are three representative examples using AO-OCT to image specific retinal tissues: (1) RNFBs, (2) foveal capillaries, and (3) cone
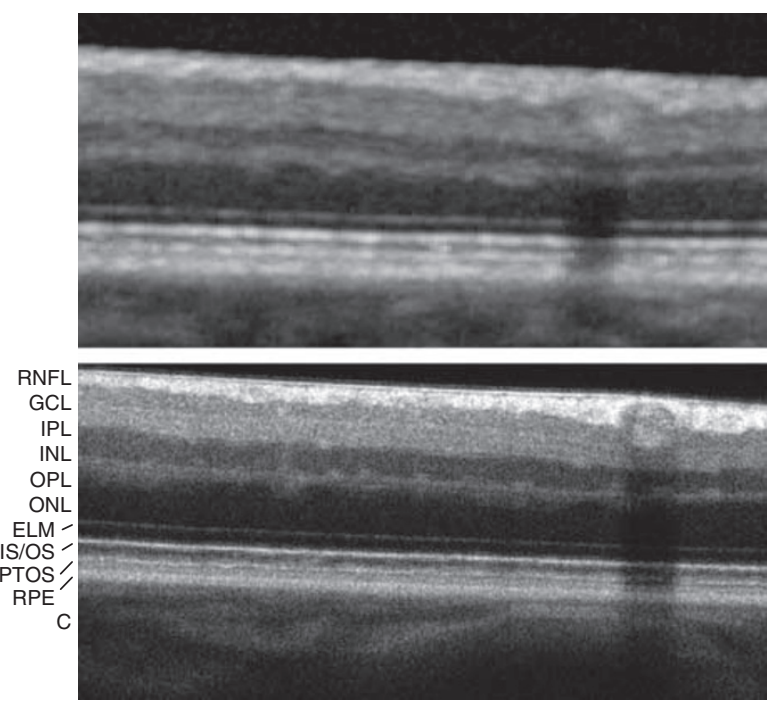

Figure 4 Comparison of B-scans acquired of the same patch of retina with the (top) Heidelberg Spectralis and (bottom) Indiana's UHR-AO-OCT instrument. B-scans are at $6^{\circ}$ superior to the fovea. B-scans subtend $3^{\circ}(900 \mu \mathrm{m})$ at the retina with the Heidelberg B-scan cropped from the much larger image in Figure 3 (white, dashed box). The large blood vessel bisecting the RNFL and GCL (top right of B-scans) is $66 \mu \mathrm{m}$ in diameter. Focus of the UHR-AO-OCT is at the RNFL. For fair comparison, both B-scans shown are an average of seven B-scans. C, choroid; ELM, external limiting membrane; GCL, ganglion cell layer; INL, inner nuclear layer; IPL, inner plexiform layer; IS/OS, inner segment/outer segment junction; ONL, outer nuclear layer; OPL, outer plexiform layer; PTOS, posterior tip of the outer segment; RNFL, retinal nerve fiber layer; RPE, retinal pigment epithelium.

photoreceptors. These highlight some of the recent work in our laboratory using UHR-AO-OCT and the measurement of specific morphological parameters: cross-sectional profile of RNFBs; diameter of foveal capillaries; and spacing and length of cone photoreceptor outer segments.

\section{Retinal nerve fiber bundles}

Early detection of axonal tissue loss is critical for managing diseases that destroy the RNFL such as glaucoma. Axonal tissue loss in the RNFL has been reported to be one of the earliest detectable glaucomatous changes, preceding morphological changes in optic nerve head and visual field loss. ${ }^{30,31}$ Fundus photography, scanning laser polarimetry, scanning laser ophthalmoscopy, and OCT have been used to analyze RNFL loss in glaucomatous eyes. ${ }^{32-35}$ Regardless of method, however, none have the necessary lateral and axial resolution to image the cross-sectional profile of individual RNFBs, as they traverse the retinal surface. Such imaging would be able to account for both 


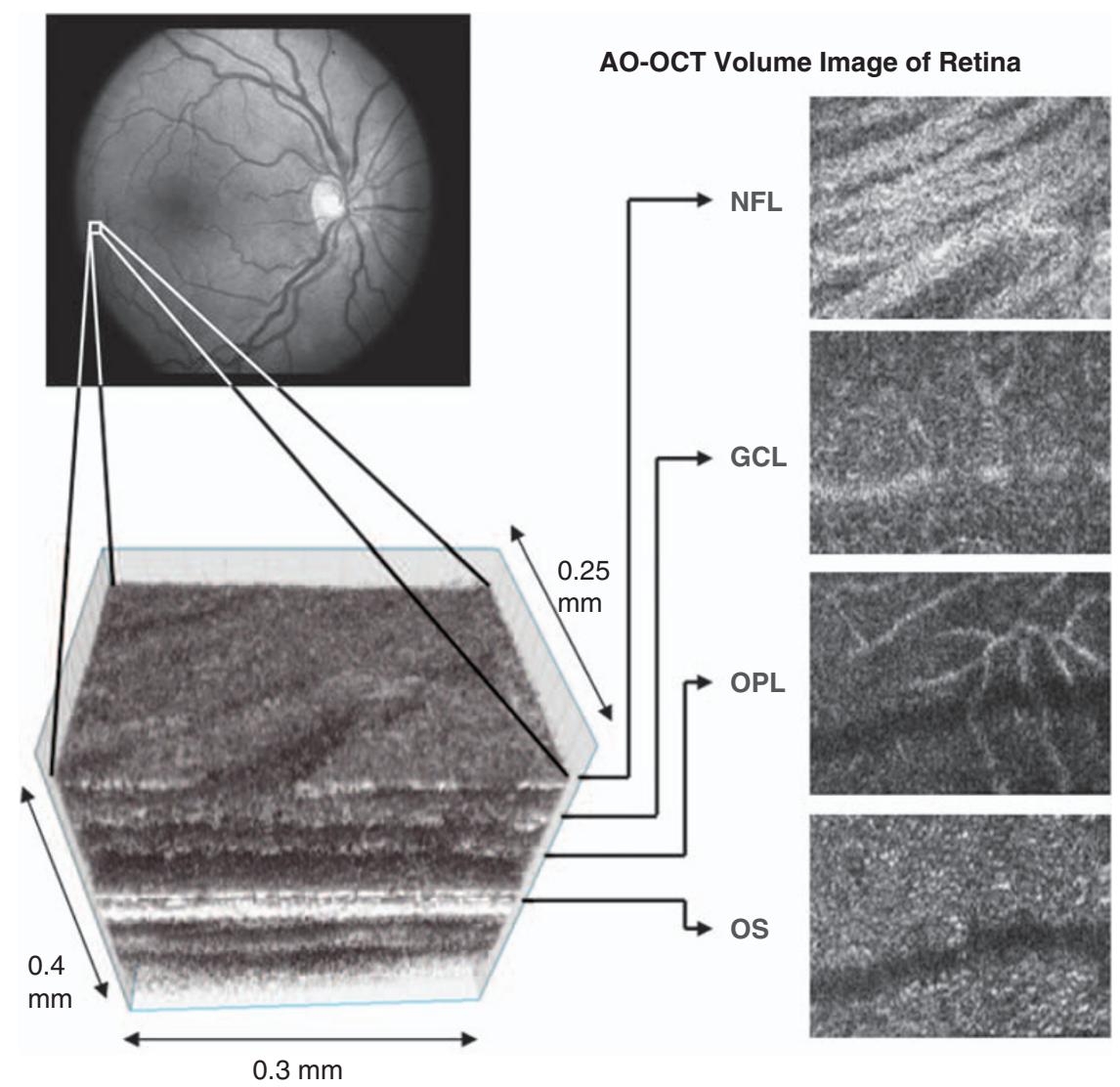

Figure 5 AO-OCT volume acquired over a $1^{\circ}$ retinal region located temporal of the fovea, as illustrated by the rectangle in the fundus photograph. The images on the right are en face views of particular retinal layers extracted from the AO-OCT volume. Retinal layers from top to bottom are: nerve fiber layer (NFL), ganglion cell layer (GCL), outer plexiform layer (OPL), and outer segment layer of photoreceptors (OS). ${ }^{29}$

thickness and width variations of individual RNFBs, and to differentiate RNFBs from the radial fibers of Müller cells that separate the bundles. This would allow extension of known morphological differences in RNFL thickness between normal, aging, and diseased retinal axonal tissue to morphological differences in RNFB area and volume.

AO-OCT represents a potential means to measure cross-sectional RNFB dimensions, owing to its micron-level 3D resolution. There have been several observational reports showing the 3D profile of RNFBs in subjects using AO-OCT. ${ }^{19,21,22}$ Although these early observations are highly encouraging, they were limited to single images acquired on single subjects with no measurements of the RNFB cross section.

To establish grounds for a population study of RNFBs, we recently imaged and measured the dimensions of RNFBs in five subjects (29-62 years of age; four clinically normal) at the same retinal eccentricities using the same UHR-AO-OCT instrument. Details of the study have been submitted to the journal Vision Research (Kocaoglu
OP, Cense B, Wang Q, Gao W, Lee S, Jonnal RS et al. Imaging retinal nerve fiber bundles using optical coherence tomography with adaptive optics). Figure 6 is a representative UHR-AO-OCT image from the study, and shows cross-sectional (B-scans) and en face (C-scan) slices extracted from the same $3^{\circ} \times 3^{\circ}$ volume of one subject. Bundle clarity (individuation) in both cross-section and en face views was found to vary with subject and retinal location, a conclusion not possible to extract from the limited AO-OCT data of RNFBs in the literature. Interestingly, the overriding determination of bundle clarity was the size of the gap between adjacent bundles rather than the size of the bundles themselves. Spacing between adjacent bundles was on the order of a few microns compared with the size of bundles that was on the order of tens of microns. The former is comparable with the resolution of the UHR-AO-OCT system $\left(3 \times 3 \times 3 \mu \mathrm{m}^{3}\right)$. Gap size at $3^{\circ}$ retinal eccentricity was found to be consistently larger than that at $6^{\circ}$, enabling the bundles to be more readily identified. Bundle 

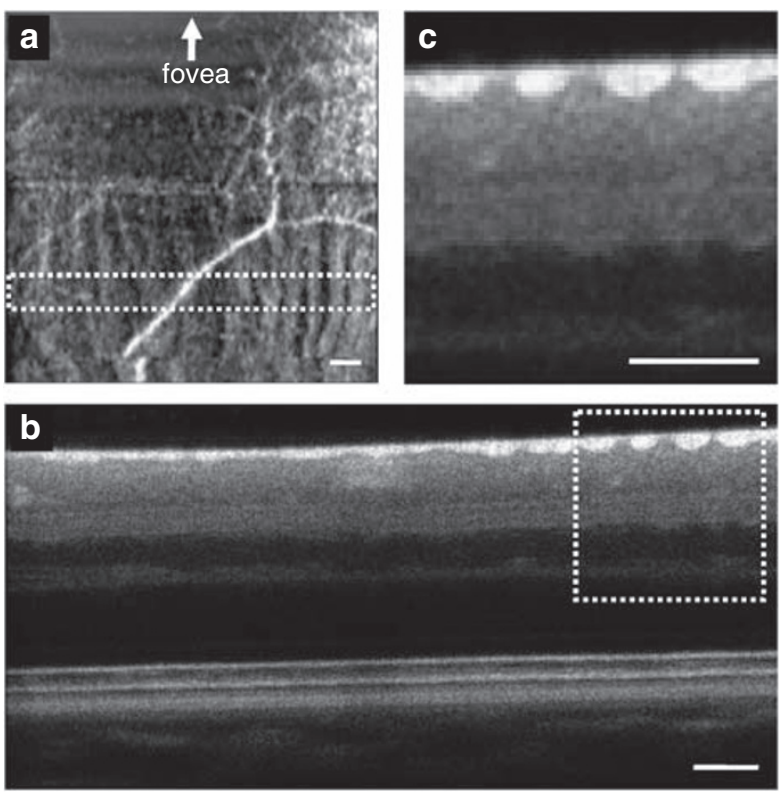

Figure 6 UHR-AO-OCT imaging of the retinal nerve fiber layer at $3^{\circ}$ nasal of the fovea. (a) En face projection $\left(3^{\circ} \times 3^{\circ}\right)$ through the RNFL reveals individual nerve fiber bundles that traverse from the optic disc to fovea (bottom to top). The bundles appear dim on a dark background. A bright capillary is superimposed on the bundles. (b) Average of eight B-scans inside the highlighted rectangle in panel a reduces speckle noise and reveals the cross-sectional profile of individual RNFBs. (c) Highlighted section in panel b is magnified two times. Scale bars denote $75 \mu \mathrm{m}$.

contrast was also critical for identifying bundles. The reflectance of RNFB tissue was found to be two times brighter $(3 \mathrm{~dB})$ than that of the Müller cells that surround the bundles (gaps). This difference provided sufficient contrast to distinguish bundle from gap when resolution allowed. In general, results of our study suggest that UHR-AO-OCT is capable of measuring the crosssectional profile of individual bundles, even at locations in which bundles are increasingly thin $(<15 \mu \mathrm{m})$. To our knowledge, these are the first measurements of RNFB cross sections reported in the living human eye.

\section{Retinal capillaries}

The retinal vasculature is a complex, layered network of vessels and capillary beds that permeate the retina.

Compromise of this support system has severe consequences to the retina and often underlies retinal pathologies, in particular diabetic retinopathy, a leading cause of blindness in the western world. Some of the earliest detectable morphological changes of this disease are microaneurysm and capillary closure. Fluorescein angiography (FA) is the gold standard for observing these fine details of the retinal vasculature and early pathological changes. ${ }^{36,37}$ Although FA is effective, it requires injection of a toxic fluorescent dye into the patient. FA also fails to detect a significant fraction of capillaries - especially small diameter ones - because of its poor depth sectioning capability and sensitivity to quality of the angiogram (optical quality of the eye). ${ }^{37}$

$\mathrm{AO}-\mathrm{OCT}$ represents a potentially attractive alternative for capillary imaging. It is non-invasive and has the necessary $3 \mathrm{D}$ resolution to map the microvasculature in all three dimensions, as, for example, to delineate the multi-laminar networks of capillaries. Many of these capillaries are only a few microns in diameter and weakly reflecting. AO has been successfully used in SLOs and flood-illumination systems to map the local 2D distribution and flow dynamics of the retinal microvasculature in living eyes. ${ }^{38-40}$ The major advantage of OCT is its substantially higher axial resolution (3-6 $\mu \mathrm{m}$ compared with $>60 \mu \mathrm{m}$ for AO-SLO and AO flood-illumination systems).

There have been numerous reported observations of AO-OCT imaging of retinal capillaries. There is also a detailed study by Hammer $e t a l^{26}$ who compared retinal capillaries in the fovea of patients with retinopathy of prematurity to that of normal individuals. Although the vasculature detail obtained with these instruments is impressive, it remains unclear the extent to which AO-OCT faithfully images capillaries, whose size varies considerably $\left(2.5-7 \mu \mathrm{m},{ }^{37} 2.5-10 \mu \mathrm{m}^{41}\right)$ and that network with vessels of increasingly larger size.

We recently addressed this concern by determining the ability of UHR-AO-OCT to image capillaries in the fovea. ${ }^{42}$ This region includes the FAZ and the network of capillaries that define its terminal rim. Use of the rim capillaries enabled us to compare the UHR-AO-OCT images with entopic viewing - an independent and established psychophysical method for mapping foveal capillaries ${ }^{43}$ on the same subjects. A representative UHR-AO-OCT en face image of the FAZ is shown in Figure 7a. By comparing with entoptic viewing on the same eyes, we showed that UHR-AO-OCT with careful focusing is indeed capable of imaging many of the capillaries proximal to the FAZ, including those of average size $(\sim 5 \mu \mathrm{m})$, as predicted by histology. This suggests that the vast majority of capillaries in the retina are likely detectable with UHR-AO-OCT, although increased instrument sensitivity may be required to offset increased scatter when the retina becomes thick and the capillaries are deep. This remains to be investigated. Aside from this caveat, our study indicates that UHR-AO-OCT is a promising option for non-invasive mapping of the intricate details of the multi-laminar networks of capillaries in the retina. In addition, the cross-sectional capability of 
UHR-AO-OCT (see Wang et $a l^{42}$ ) provides quantitative depth information of the various multi-laminar networks of capillaries.

Future technological advances, in particular high-speed OCT imaging and phase detection, ${ }^{44}$ will only improve on UHR-AO-OCT's capability to image retinal capillaries and detect some of the earliest morphological changes associated with vasculature disease such as diabetic retinopathy. With the Indiana UHR-AO-OCT system, we have recently implemented ultrahigh-speed imaging, which has allowed finer sampling of the retina while keeping volume acquisition time constant. An example image of the FAZ at this higher speed is shown in Figure $7 \mathrm{~b}$. Comparison of Figures $7 \mathrm{a}$ and $\mathrm{b}$ shows that the finer sampling noticeably improves visibility and sharpness of the small capillaries.

\section{Photoreceptors}

Cone photoreceptors undergo degeneration and death with numerous retinal diseases, including ARMD and retinal dystrophies such are retinitis pigmentosa. Although a great deal has been learned about these diseases through histological studies, the need to destroy the tissue and artifacts generated during the preparation confound interpretation. AO-SLOs and AO floodillumination systems offer a non-invasive alternative, providing en face views of individual cones for monitoring disruptions in their packing and brightness that are known to occur with disease progression. ${ }^{45-47}$ AO-OCT represents a third alternative that-although less mature than AO-SLO and AO flood-illumination
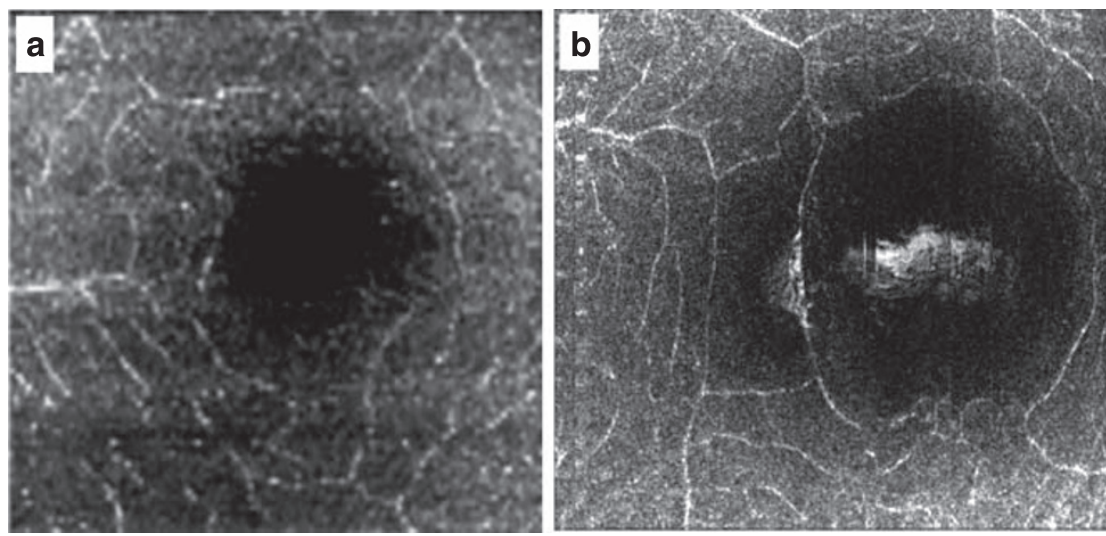

Figure 7 UHR-AO-OCT imaging of the retinal capillaries proximal to the foveal avascular zone. En face projections $\left(3^{\circ} \times 3^{\circ}\right)$ through the multi-laminar networks of capillaries of the foveal region are shown in two normal subjects. A well-defined FAZ is evident in both panels $a$ and $b$. Volumes were acquired with UHR-AO-OCT using A-scan rates of (a) $22.5 \mathrm{KHz}$ (comparable with commercial OCT rates) and (b) $125 \mathrm{KHz}$. Both were acquired in $4.5 \mathrm{~s}$, but the latter sampled the retina 5.6 times finer, which noticeably improves the visibility and sharpness of the small capillaries. AO adjusted focus to optimize vessel clarity. Central bright spot in panel $\mathrm{b}$ is a residual of the fovea reflex that was not completely removed in post processing when the capillary sub-region was extracted.
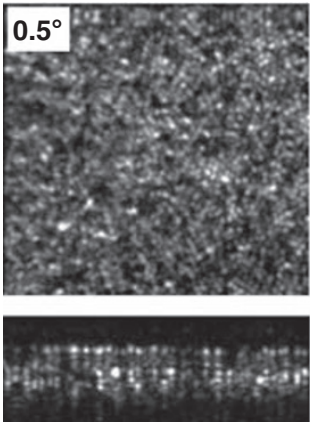
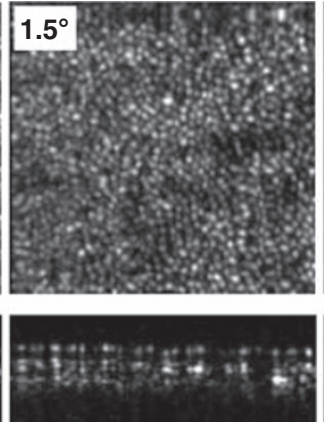
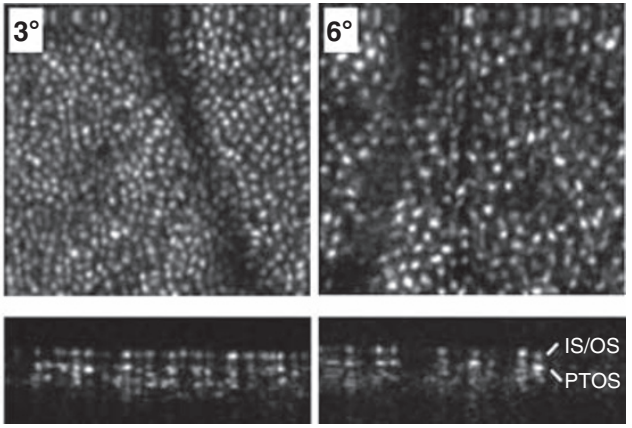

Figure 8 UHR-AO-OCT imaging of cone photoreceptors. En face projections (top) and cross-sections of cone photoreceptors (bottom) are shown at $0.5^{\circ}, 1.5^{\circ}, 3^{\circ}$, and $6^{\circ}$ temporal to the fovea of a 24-year-old normal subject. Projections are $0.6^{\circ} \times 0.6^{\circ}$ and B-scans are $0.6^{\circ}$ wide. A-scans were acquired at $167 \mathrm{KHz}$, six to eight times faster than that of commercial OCT. Cross-sectional images are single B-scans extracted near the center of the en face projections. Cone spacing increases with retinal eccentricity and is consistent with that reported in the literature. ${ }^{50,51}$ 
systems - holds the greatest potential to extend en face imaging to 3D imaging of cone morphology.

In recent years, cone photoreceptors have been imaged with a variety of OCT configurations: OCT, ${ }^{48,49}$ AO-OCT, ${ }^{15,17}$ and UHR-AO-OCT. ${ }^{19-22}$ OCT studies rely primarily on high-speed imaging to minimize motion artifacts, but without $\mathrm{AO}$, they are limited to eccentricities relatively distant from the fovea (greater than $\sim 4^{\circ}$ ), where cones are comparatively large and widely spaced, and eyes endowed with good optics. This strategy follows that used for the first observations of individual cones in the living human retina. ${ }^{52}$ AO-OCT does not share these limitations and has been used in early studies of the 3D structure of cones. More detailed and exhaustive studies, however, have largely been impractical because of eye motion artifacts that prevent tracking of individual cones across frames of AO-OCT videos.

We recently addressed this technical bottleneck, or at least sufficiently so, to demonstrate cone tracking and imaging in three dimensions and in time using UHR-AO-OCT. ${ }^{53,54}$ This was accomplished by high-speed image acquisition (up to $200 \mathrm{KHz}$ ) using a state-of-the-art high-speed CMOS detector (to reduce the deleterious effects of eye motion on volumetric images) and a novel post-processing registration/dewarping methodology (to further reduce eye motion effects). The combination of the two reduced eye motion to less than a fraction of a cone width. With this level of motion compensation, we were able to track individual cones over periods as long as 10 days. This time duration is more than adequate for studying the physiological processes of disc renewal and phagocytosis at the individual cone level, processes that are known to be disrupted by retinal disease such as ARMD and retinitis pigmentosa. We were also able to image cones as close as $0.5^{\circ}$ from the fovea (the most critical area of patient vision), which is the densest packing of cones observed to date with AO-OCT. Figure 8 shows representative en face and cross-sectional views of individual cones acquired with the high-speed UHR-AO-OCT system. Note the level of cone detail at the four retinal eccentricities and in both views, as, for example, the decrease in cone outer segment length with retinal eccentricity. To our knowledge, this is the first demonstration of $4 \mathrm{D}$ imaging and tracking of cones using AO-OCT.

\section{Conclusion}

$\mathrm{AO}-\mathrm{OCT}$ is a maturing technology that has experienced tremendous technological advances since its first reporting 8 years ago. ${ }^{9}$ The technical benefits of adding AO to OCT significantly increase the imaging capability of OCT in ways that make it well suited for 3D cellular imaging in the retina. Here, we presented representative examples of cellular structures that can be visualized with AO-OCT. Results from three studies from our laboratory indicate that UHR-AO-OCT is capable of measuring the cross-sectional profiles of individual RNFBs the diameters of retinal capillaries that define the terminal rim of the FAZ; and the spacing and length of cone photoreceptor outer segments as close as $0.5^{\circ}$ from the fovea center.

\section{Conflict of interest}

Donald Miller is co-inventor of intellectual property related to the combination of $\mathrm{AO}$ and OCT.

\section{Acknowledgements}

This study was financially supported, in part, by the National Eye Institute grants 1R01EY018339, 5R01 EY014743, and P30 EY019008.

\section{References}

1 Huang D, Swanson EA, Lin CP, Schuman JS, Stinson WG, Chang W et al. Optical coherence tomography. Science 1991; 254: 1178-1181.

2 Drexler W, Fujimoto JG (eds) Optical Coherence Tomography Technology and Applications. Springer-Verlag: New York, 2008.

3 Liang J, Williams DR, Miller DT. Supernormal vision and high resolution retinal imaging through adaptive optics. J Opt Soc Am A 1997; 14: 2884-2892.

4 Porter J, Queener H, Lin J, Thorn K, Awwal A (eds) Adaptive Optics for Vision Science: Principles, Practices, Design and Applications. Wiley: New York, 2006.

5 Miller DT, Roorda A. Adaptive optics in retinal microscopy and vision. In: Bass M, Enoch JM, Lakshminarayanan V (eds). Handbook of Optics Volume III. McGraw Hill: New York, 2009 pp 15.1-15.30.

6 Dreher AW, Bille JF, Weinreb RN. Active optical depth resolution improvement of the laser tomographic scanner. Appl Opt 1989; 24: 804-808.

7 Roorda A, Romero-Borja F, Donnelly WJ, Queener H, Hebert TJ, Campbell MCW. Adaptive optics scanning laser ophthalmoscopy. Opt Express 2002; 10: 405-412.

8 Wojtkowski M. High-speed optical coherence tomography: basics and applications. Appl Opt 2010; 49: D30-D61.

9 Miller DT, Qu J, Jonnal RS, Thorn K. Coherence gating and adaptive optics in the eye. In: Valery V, Tuchin V, Izatt JA, Fujimoto JG (eds). Coherence Domain Optical Methods and Optical Coherence Tomography in Biomedicine VII. SPIE Optical Engineering Press: Bellingham, 2003 pp 65-72.

10 Hermann B, Fernandez EJ, Unterhuber A, Sattmann H, Fercher AF, Drexler W et al. Adaptive-optics ultrahighresolution optical coherence tomography. Opt Lett 2004; 29: 2142-2144.

11 Merino D, Dainty C, Bradu A, Podoleanu AG. Adaptive optics enhanced simultaneous en-face optical coherence tomography and scanning laser ophthalmoscopy. Opt Express 2006; 14: 3345-3353. 
12 Pircher M, Zawadzki RJ, Evans JW, Werner JS, Hitzenberger CK. Simultaneous imaging of human cone mosaic with adaptive optics enhanced scanning laser ophthalmoscopy and high-speed transversal scanning optical coherence tomography. Opt Lett 2008; 33: 22-24.

13 Zhang Y, Rha J, Jonnal RS, Miller DT. Adaptive optics parallel spectral domain optical coherence tomography for imaging the living retina. Opt Express 2005; 13: 4792-4811.

14 Zawadzki RJ, Jones S, Olivier SS, Zhao M, Bower BA, Izatt JA et al. Adaptive-optics optical coherence tomography for high-resolution and high-speed 3D retinal in vivo imaging. Opt Express 2005; 13: 8532-8546.

15 Zhang Y, Cense B, Rha J, Jonnal RS, Gao W, Zawadzki RJ et al. High-speed volumetric imaging of cone photoreceptors with adaptive optics spectral domain optical coherence tomography. Opt Express 2006; 14: 4380-4394.

16 Bigelow CE, Iftimia NV, Ferguson RD, Ustun TE, Bloom B, Hammer DX. Compact multimodal adaptive-optics spectral-domain optical coherence tomography instrument for retinal imaging. J Opt Soc Am A 2007; 24: 1327-1336.

17 Zawadzki RJ, Choi SS, Jones SM, Oliver SS, Werner JS. Adaptive optics-optical coherence tomography: optimizing visualization of microscopic retinal structures in three dimensions. J Opt Soc Am A 2007; 24: 1373-1383.

18 Fernández EJ, Považay B, Hermann B, Unterhuber A, Sattmann H, Prieto PM et al. Three-dimensional adaptive optics ultrahigh-resolution optical coherence tomography using a liquid crystal spatial light modulator. Vision Res 2005; 45: 3432-3444.

19 Zawadzki RJ, Cense B, Zhang Y, Choi SS, Miller DT, Werner JS. Ultrahigh-resolution optical coherence tomography with monochromatic and chromatic aberration correction. Opt Express 2008; 16: 8126-8143.

20 Fernández EJ, Hermann B, Považay B, Unterhuber A, Sattmann H, Hofer B et al. Ultrahigh resolution optical coherence tomography and pancorrection for cellular imaging of the living human retina. Opt Express 2008; 16: 11083-11094.

21 Cense B, Koperda E, Brown JM, Kocaoglu OP, Gao W, Jonnal RS et al. Volumetric retinal imaging with ultrahigh-resolution spectral-domain optical coherence tomography and adaptive optics using two broadband light sources. Opt Express 2009; 17: 4095-4111.

22 Torti C, Považay B, Hofer B, Unterhuber A, Carroll J, Ahnelt PK et al. Adaptive optics optical coherence tomography at $120000 \mathrm{depth}$ scans/s for non-invasive cellular phenotyping of the living human retina. Opt Express 2009; 17: 19382-19400.

23 Mujat M, Ferguson RD, Patel AH, Iftimia N, Lue N, Hammer DX. High resolution multimodal clinical ophthalmic imaging system. Opt Express 2010; 18: 11607-11621.

24 Cense B, Gao W, Brown JM, Jones SM, Jonnal RS, Mujat M et al. Retinal imaging with polarization-sensitive optical coherence tomography and adaptive optics. Opt Express 2009; 17: 21634-21651.

25 Boycott BB, Dowling JE. Organization of the primate retina: light microscopy. Philos Trans R Soc Lond B 1969; 255: 109-184.

26 Hammer DX, Iftimia NV, Ferguson RD, Bigelow CE, Ustun TE, Barnaby AM et al. Foveal fine structure in retinopathy of prematurity: an adaptive optics Fourier domain optical coherence tomography study. Invest Ophthalmol Vis Sci 2008; 49: 2061-2070.
27 Choi SS, Zawadzki RJ, Keltner JL, Werner JS. Changes in cellular structures revealed by ultra-high resolution retinal imaging in optic neuropathies. Invest Ophthalmol Vis Sci 2008; 49: 2103-2119.

28 Choi SS, Zawadzki RJ, Lim MC, Brandt JD, Keltner JL, Doble $\mathrm{N}$ et al. Evidence of outer retinal changes in glaucoma patients as revealed by ultrahigh-resolution in vivo retinal imaging. Br J Ophthalmol 2011; 95(1): 131-141.

29 Zawadzki RJ, Zhang Y, Jones SM, Choi SS, Cense B, Chen D et al. Application of adaptive optics: optical coherence tomography for in vivo imaging of microscopic structures in the retina and optic nerve head. In: Manns F, Soederberg PG, Ho A, Stuck BE, Belkin M (eds). Ophthalmic Technologies XVII. SPIE Optical Engineering Press: Bellingham, 2007 pp 642610-1-642610-8.

30 Sommer A, Miller NR, Pollack I, Maumenee AE, George T. The nerve fiber layer in the diagnosis of glaucoma. Arch Ophthalmol 1977; 95: 2149-2156.

31 Sommer A, Katz J, Quigley HA, Miller NR, Robin AL, Richter RC et al. Clinically detectable nerve fiber atrophy precedes the onset of glaucomatous field loss. Arch Ophthalmol 1991; 109: 77-83.

32 Hoyt WF, Frisen L, Newman NM. Fundoscopy of nerve fiber layer defects in glaucoma. Invest Ophthalmol Vis Sci 1973; 12: 814-829.

33 Medeiros FA, Alencar LM, Zangwill LM, Bowd C, Vizzeri G, Sample PA et al. Detection of progressive retinal nerve fiber layer loss in glaucoma using scanning laser polarimetry with variable corneal compensation. Invest Ophthalmol Vis Sci 2009; 50: 1675-1681.

34 Caprioli J, Park HJ, Ugurlu S, Hoffman D. Slope of the peripapillary nerve fiber layer surface in glaucoma. Invest Ophthalmol Vis Sci 1998; 39: 2321-2328.

35 Seong M, Sung KR, Choi EH, Kang SY, Cho JW, Um TW et al. Macular and peripapillary retinal nerve fiber layer measurements by spectral domain optical coherence tomography in Normal-Tension Glaucoma. Invest Ophthalmol Vis Sci 2010; 51: 1446-1452.

36 Arend O, Wolf S, Jung F, Bertram B, Pöstgens H, Toonen H et al. Retinal microcirculation in patients with diabetes mellitus: dynamic and morphological analysis of perifoveal capillary network. Br J Ophthalmol 1991; 75: 514-518.

37 Weinhausa RS, Burkea JM, Delori FC, Snodderly DM. Comparison of fluorescein angiography with microvascular anatomy of macaque retinas. Exp Eye Res 1995; 61: 1-16.

38 Martin JA, Roorda A. Direct and noninvasive assessment of parafoveal capillary leukocyte velocity. Ophthalmology 2005; 112: 2219-2224.

39 Rha J, Jonnal RS, Thorn KE, Qu J, Zhang Y, Miller DT. Adaptive optics flood-illumination camera for high speed retinal imaging. Opt Express 2006; 14: 4552-4569.

40 Zhang Z, Petrig BL, Qi X, Burns SA. In vivo measurement of erythrocyte velocity and retinal blood flow using adaptive optics scanning laser ophthalmoscopy. Opt Express 2008; 16: 12746-12756.

41 Snodderly DM, Weinhaus RS, Choi JC. Neural-vascular relationships in central retina of Macaque monkeys (Macaca fascicularis). J Neurosci 1992; 12: 1169-1193.

42 Wang Q, Kocaoglu OP, Cense B, Bruestle J, Jonnal RS, Gao $\mathrm{W}$ et al. Imaging retinal capillaries using ultrahigh-resolution optical coherence tomography and adaptive optics. Invest Ophthalmol Vis Sci, E-pub ahead of print 18 January 2011; doi:10.1167/iovs. $10-6424$. 
43 Bradley A, Applegate RA, Zeffren BS, van Heuven WAJ. Psychophysical measurement of the size and shape of the human foveal avascular zone. Ophthal Physiol Opt 1992; 12: 18-23.

44 Fingler J, Zawadzki RJ, Werner JS, Schwartz D, Fraser SE. Volumetric microvascular imaging of human retina using optical coherence tomography with a novel motion contrast technique. Opt Express 2009; 17: 22190-22200.

45 Choi SS, Doble N, Hardy JL, Steven MJ, Keltner JL, Oliver SS et al. In vivo imaging of the photoreceptor mosaic in retinal dystrophies and correlations with visual function. Invest Ophthalmol Vis Sci 2006; 47: 2080-2092.

46 Wolfing JI, Chung M, Carroll J, Roorda A, Williams DR. High resolution retinal imaging of cone-rod dystrophy. Ophthalmology 2006; 113: 1014-1019.

47 Duncan JL, Zhang Y, Gandhi J, Nakanishi C, Othman M, Branham $\mathrm{KEH}$ et al. High-resolution imaging with adaptive optics in patients with inherited retinal degeneration. Invest Ophthalmol Vis Sci 2007; 48: 3283-3291.

48 Pircher M, Baumann B, Götzinger E, Sattmann H, Hitzenberger CK. Simultaneous SLO/OCT imaging of the human retina with axial eye motion correction. Opt Express 2007; 15: 16922-16932.
49 Potsaid B, Gorczynska I, Srinivasan VJ, Chen Y, Jiang J, Cable A et al. Ultrahigh speed spectral/Fourier domain OCT ophthalmic imaging at 70,000-312,500 axial scans per second. Opt Express 2008; 16: 15149-15169.

50 Curcio CA, Sloan KR, Packer O, Hendrickson AE, Kalina RE. Distribution of cones in human and monkey retina: individual variability and radial asymmetry. Science 1987; 236: 579-582.

51 Chui TY, Song H, Burns SA. Individual variations in human cone photoreceptor packing density: variations with refractive error. Invest Ophthalmol Vis Sci 2008; 49: 4679-4687.

52 Miller DT, Williams DR, Morris GM, Liang J. Images of cone photoreceptors in the living human eye. Vision Res 1996; 36: 1067-1079.

53 Kocaoglu OP, Lee S, Jonnal RS, Wang Q, Herde AE, Derby JC et al. Imaging cone photoreceptors in three dimensions and in time using ultrahigh resolution optical coherence tomography with adaptive optics. Biomed Opt Express (Accepted).

54 Kocaoglu OP, Lee S, Jonnal RS, Wang Q, Herde AE, Besecker J et al. 3D imaging of cone photoreceptors over extended time periods using optical coherence tomography with adaptive optics. Proc SPIE 2011; 7885, 7885-10. 